\title{
Exploring the Use of Landmarks for Mobile Navigation Support in Natural Environments
}

\author{
Caroline Snowdon \\ School of Computing Science \\ University of Newcastle Upon Tyne, UK \\ callisnowdon@hotmail.co.uk
}

\author{
Christian Kray \\ School of Computing Science \\ University of Newcastle Upon Tyne, UK \\ c.kray@ncl.ac.uk
}

\begin{abstract}
Landmarks are a key element in navigation and have been used extensively to provide navigation support to pedestrians through mobile devices in urban areas. Natural environments differ significantly from built environments in a number of ways including, for example, the degree of structure and regularity, the types and density of landmarks, and the way in which people navigate in those environments. In this paper, we investigate how people currently navigate 'in the wild', and whether landmark-based navigation support through mobile devices is a feasible option in such settings. We present results from two studies: a questionnaire-based study focussing on current practice and the use of landmarks, and a qualitative lab-based study using immersive panoramic photographs and photographs of (natural) landmarks investigating the feasibility of landmark-based navigation support in natural environments. The results indicate that a small number of means are currently used for navigation in the wild, and that certain types of landmarks might be feasible for navigation support on mobile devices. We also found initial evidence that immersive panoramic photographs may constitute a promising way to evaluate mobile applications.
\end{abstract}

\section{Categories and Subject Descriptors}

H.5.1 [Information Interfaces and Presentation]: Multimedia Information Systems - artificial, augmented and virtual realities; H.5.2 [Information Interfaces and Presentation]: User Interfaces-graphical user interfaces

\section{General Terms}

Design, Experimentation, Human Factors

\section{Keywords}

mobile guides, landmarks, navigation, user studies, natural environments
Copyright is held by the author/owner(s).

Mobile HCI 2009, September 15-18, 2009 Bonn, Germany

ACM 978-1-60558-281-8/09/09.

\section{INTRODUCTION}

Mobile guides are among the most widely used location based services (LBS). Navigation support is available for cars, motorbikes and bicycles as well as for pedestrians. Systems providing guidance for pedestrians have been developed to help people find their way in different environments such as urban areas, museums and trade fairs. Some research prototypes exist that seamlessly integrate outdoor and indoor environments, or that link the use of public transport and walking directions. The vast majority of existing commercial and research systems provide navigation support in the built environment but comparatively little work has been done for natural environments. Obviously, manmade and natural areas differ significantly in a number of ways (e.g. degree of structure or repetitiveness, type and number of landmarks, provision of signage, infrastructure, density of people). Consequently, navigation in both types of environments will differ as well.

In this paper, we are investigating how people currently navigate natural environments and which tools they use. In particular, we are interested in the role landmarks play and whether a mobile guide based on landmarks would be suitable for navigation in the wild. The following section discusses related work and analyses why landmark-based navigation support on mobile devices might be well suited for natural environments (section 2). We will then describe the two studies we carried out to investigate navigation support in the wild: a questionnaire-based study focussing on current practice (section 3) and a lab-based immersive panorama study testing the use of landmarks for mobile guides (section 4). In section 5, we discuss the findings of both studies and point out implications for mobile navigation support in the wild. We conclude by summarising the key contributions and pointing out further research opportunities.

\section{RELATED WORK}

Previous work on mobile guides both in academia and for commercial applications (see [2] for a survey) mainly focusses on systems supporting pedestrians in urban environments. Frequently, these systems relies on the use of landmarks for navigation support. Previous research has identified how people use landmarks when navigating, which features are required to make an object suitable for use as a landmark, and how mobile guides can incorporate landmarks to generate route directions (cf. e.g. $[14,12])$ The GUIDE system [5] for example made use of photographs of landmarks to help people localise themselves and to convey directions. More recent guide applications for mobile 
phones (cf. e.g. [13, 1]) benefit from additional sensors such as (A)GPS, increased computational power and online mapping services. Nevertheless, they make extensive use of landmarks. In addition to urban outdoor environments, research on mobile guides has been extended to indoor areas [4], where landmarks also play an important role in describing routes [10]. Since indoor environments often are quite homogeneous and symmetrical, the use of 'artificial/personal landmarks' has also been explored [17]. Our work differs from these systems in two ways: we focus on natural rather than man-made landmarks, and their use for mobile navigation support in natural rather than urban environments.

Using landmarks is just one of several ways to convey directions but they are frequently used in the context of textual, pictorial and auditory instructions [11]. Using photographs of landmarks has been shown to be a viable way to provide navigation support [5], in particular to older people [8], who are potentially more likely to experience difficulties in perceiving and understanding complex instructions given through a mobile device with a small screen. Davies et al. [6] recently looked into the use of photos (of landmarks) and image recognition to support people exploring urban areas. Although they concluded that image recognition is not yet feasible, the general approach of using photographs shared by anyone to support situated activities is promising. Our work differs from this approach by not requiring automatic image recognition (we rely on recognition through a human user), by focussing on navigation and by investigating the use of landmarks in natural rather than urban environments.

While there is a large body of work on mobile guides for urban areas, comparatively little research has been published on this type of system for use in natural environments. The Ambient Woods project [15] investigated the use of mobile guides 'in the wild' for educational purposes. Several prototypes were developed and tested with school children, aged 11 to 12 ; although navigation was implicitly part of the tasks given to the children, the actual system was more focussed on facilitating learning than giving directions. Although not focussed on natural environments, field test for the AudioGPS system [9] were carried out in such settings. Bidwell et al. have investigated the types of landmarks people use when navigating areas with a mixture of natural and 'urban' features [3]. One outcome from their studies was the need to include additional landmarks beside objects that stand out from their environment (e. g. landmarks located at decision points and well suited for giving directions). Both studies presented later on in this paper extend this work by identifying what natural landmarks may be well suited for navigation and by testing such landmarks with participants in a simulated natural environment. Düpmeier and Ruchter [7] carried out a 'field' study on interface design for mobile guides that are meant to be used in natural environments. One of their main findings was the importance of testing interfaces for use in natural environments in situ, which can be logistically challenging. The approach we introduce in Section 4 provides a means to considerably simplify such tests by 'recreating' natural environments in the lab.

Mobile guides such as the ones discussed above can provide navigation support in many ways including, for example, audio instructions, (annotated) maps, or tactile output (cf.e.g. [11]). In principle, all these means are also applicable to natural environments but there are several reasons why using landmarks for navigation support on mobile de- vices could be particularly effective in natural environments.

Natural environments are less regularly and tightly structured than urban areas: the latter possess a street network, which many mobile guides for pedestrians rely on heavily, the former does not. Landmarks, however, are present in both kinds of environments, and could thus be used for navigation support 'in the wild'. Furthermore, landmarks in natural environments often are highly visible (e. g. peaks, lakes, or particular features of the landscape), whereas a blocked line of sight can frequently be an issue in urban areas. Since the enjoyment of nature is often one of the main reasons for venturing into the countryside, the use of landmarks for directions may reduce the frequency at which a mobile guide would have to be consulted and therefore would interfere less with people's experience. Finally, photographing landmarks is often part of the activities people perform while they are in natural environments anyway. Consequently, it is likely that a significant number of community generated photographs of landmarks in natural environments would be readily available for use in mobile guide.

Based on these considerations, we concluded that it would make sense to further investigate landmark-based navigation support in natural environments on mobile devices. In the following, we first present a questionnaire-based study aimed at establishing current practice and then report on a labstudy testing the use of landmarks on mobile devices for navigation support in the wild.

\section{STUDY 1: CURRENT PRACTICE}

In order to find out how people currently navigate in natural environments (e.g. what technology they use and how they use it), we designed an online questionnaire that we distributed widely, in particular to societies and groups who regularly engage in activities such as fell walking and cross country hiking. In the following, we describe the design of the questionnaire, how it was disseminated and the results we obtained. The outcomes are discussed in more detail in section 5 alongside the results of the second study.

\subsection{Questionnaire Design}

In order to ensure a large number of responses, we designed the questionnaire so that people would be able to answer all questions quickly (in about 15 minutes). We also split the questions into five parts, each of which fitted onto a single web page and could be answered in a few minutes. Each part had to be filled in completely before participants were allowed to proceed to subsequent parts. The first part related to how often people spent time in the countryside and to the activities they pursued. The second part was focussed on the methods used for navigation used and what their perceived advantages and disadvantages were. If respondents had selected any of the GPS options in part two they were presented with module three, which related to the reasons for choosing a particular GPS device, the features it had and any features that they felt were lacking on the device. In part four the questions centred around natural landmarks and what data respondents use when making navigational decisions. The final part of the questionnaire was designed to collect basic demographic data, such as the age range of the respondents and their confidence when using technology. In total, participants had to answer 28 questions; five of those were open-ended question, while the remaining 23 were either single- or multiple-choice questions. 


\subsection{Procedure}

We used a commercial web service (www.esurveypro.com) to host the questionnaire and collect the responses from participants. The link to the questionnaire was sent to several individuals as well as to a number of organisations and mailing lists known to be frequented by people who regularly explore natural environments (e.g. fell walking societies, cross country hikers, orienteering clubs). Some of these organisations also displayed the link on their web site. The questionnaire was open for three weeks during June and July 2008. The survey data returned by the web service was provided in an aggregate form, which precluded certain types on analysis. In particular, it was not possible to extract individual answers and we were thus unable to analyse the results according to gender or age.

\subsection{Results}

During the time the questionnaire was open, 285 people filled in at least one of the five pages, and 179 completed the whole survey. The first set of questions was aimed at gauging how much time people spent in the countryside and which activities they engaged with. Almost 50\% reported visiting the countryside at least once a week (48\%, 133 replies), $35 \%$ said they visit the countryside at least once a month (98 replies) and a further $9 \%$ replied they did so once every three months (25 replies). Only $8 \%$ of the participants reported visiting the countryside less than twice a year $(22$ replies). Thus, of the 278 people replying to this question, more than $80 \%$ venture into natural environments at least once a month.

Table 1: Dominant and occasional activities participants engaged with while in the countryside (total number of responses (Cnt) and percentages (Pctge) - multiple replies permitted for occasional activities).

\begin{tabular}{|l|r|r||r|r|}
\hline & \multicolumn{2}{|c||}{ Dominant } & \multicolumn{2}{c|}{ Occasional } \\
\hline Activity & Cnt & Pctge & Cnt & Pctge \\
\hline Mountaineering & 21 & $8 \%$ & 81 & $11 \%$ \\
\hline Fell/Hill Walking & 63 & $23 \%$ & 96 & $13 \%$ \\
\hline Rambling/Walking & 126 & $45 \%$ & 79 & $11 \%$ \\
\hline Guided Walks & 2 & $1 \%$ & 88 & $12 \%$ \\
\hline Orienteering & 0 & $0 \%$ & 73 & $10 \%$ \\
\hline Mountain Biking & 6 & $2 \%$ & 67 & $9 \%$ \\
\hline Horse Riding & 5 & $2 \%$ & 78 & $11 \%$ \\
\hline Geo-Caching & 17 & $6 \%$ & 78 & $11 \%$ \\
\hline Fell Running & 13 & $5 \%$ & 19 & $3 \%$ \\
\hline (Rock) Climbing & 11 & $4 \%$ & 23 & $3 \%$ \\
\hline Other & 15 & $5 \%$ & 41 & $6 \%$ \\
\hline Sum & $\mathbf{2 7 9}$ & $\mathbf{1 0 0 \%}$ & $\mathbf{7 2 3}$ & $\mathbf{1 0 0 \%}$ \\
\hline
\end{tabular}

The next two questions were aimed at identifying which activities participants engage with while they are in the countryside. Figure 1 and Table 1 summarise the main findings. When asked about which activities participants take part in occasionally, responses were fairly evenly distributed (except for fell running and climbing, which were less popular). However, more than two thirds of the participants identified walking (fell/hill walking/rambling) as their main activity.

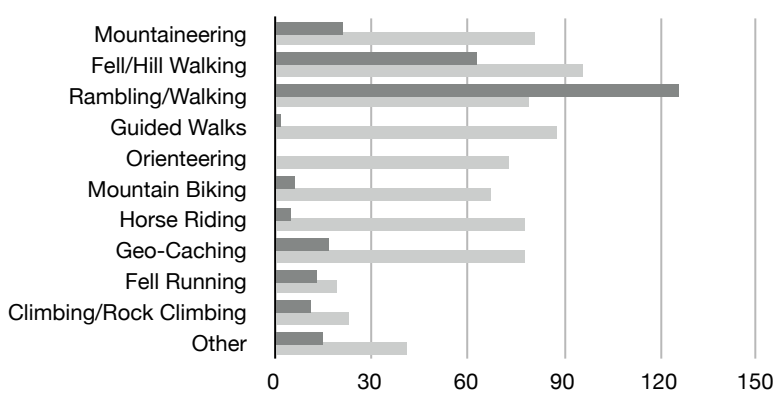

Figure 1: Dominant (dark bars) and occasional (light bars) activities participants engaged with while in the countryside (number of responses).

The second set of questions was aimed at finding out more about how people navigate while in the countryside by asking which method participants use (occasionally and predominantly). Figure 2 and Table 2 summarise the findings for the methods people used predominantly and occasionally. More than half of the participants mainly relied on a map (with or without a compass) for navigation, while a further $22 \%$ listed following a path or trail as their main method of navigating the countryside. Dedicated GPS tools were used by $11 \%$ while the use of GPS on mobile phones was the main mode of navigation for only $3 \%$ of the participants. When asked which methods they had used occasionally, the distribution of replies closely mirrored the responses we had obtained when asking for the preferred means of navigation.

Table 2: Dominant and occasional activities participants engaged with while in the countryside (total number of responses (Cnt) and percentages (Pctge) - multiple replies permitted for occasional use).

\begin{tabular}{|l|r|r||r|r|}
\hline & \multicolumn{2}{|c||}{ Dominant } & Occasional \\
\hline Method & Cnt & Pctge & Cnt & Pctge \\
\hline Follow a group leader & 9 & $4 \%$ & 33 & $6 \%$ \\
\hline Use a map & 56 & $28 \%$ & 146 & $26 \%$ \\
\hline Map and compass & 57 & $28 \%$ & 136 & $24 \%$ \\
\hline GPS tool & 22 & $11 \%$ & 69 & $12 \%$ \\
\hline GPS on mobile phone & 3 & $1 \%$ & 11 & $2 \%$ \\
\hline Follow path/trail & 45 & $22 \%$ & 145 & $26 \%$ \\
\hline Local/prev. knowledge & 3 & $1 \%$ & 18 & $3 \%$ \\
\hline Other & 6 & $3 \%$ & 18 & $3 \%$ \\
\hline Sum & $\mathbf{2 0 1}$ & $\mathbf{1 0 0 \%}$ & $\mathbf{5 6 6}$ & $\mathbf{1 0 0 \%}$ \\
\hline
\end{tabular}

We also asked participants how long they have been using their preferred navigation method. The vast majority of people $(81 \%, 163$ responses) reported to have been using their method of choice for more than five years. Another $15 \%$ (30 replies) reported having used it for between one and five years and $3 \%$ ( 7 replies) said between half a year and a year. Of the 201 responses, only one person reported less than a month of usage for the preferred navigation method. Hence, almost all participants had not changed their method of choice in the last six months and the majority had not changed it in the last five years. 


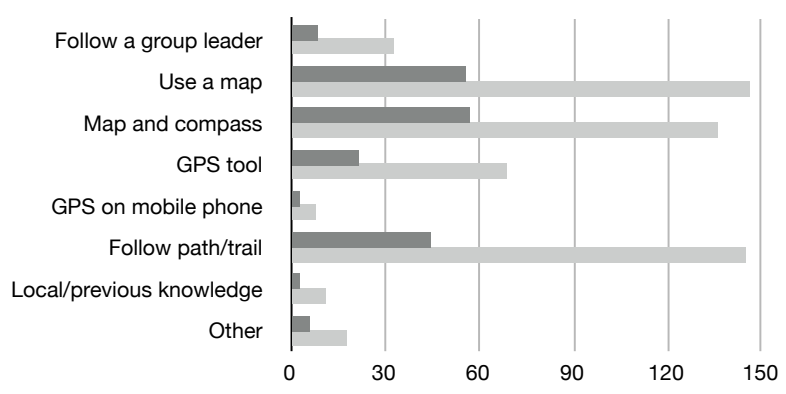

Figure 2: Dominant (dark bars) and occasional (light bars) methods/tools used for navigation while in the countryside (number of responses).

Only those participants who had used a GPS-enabled device before (as preferred mode of navigation or occasionally) were presented with the third set of questions. The first question in this part was meant to identify features people are looking for in a GPS device (multiple responses allowed). The results did not reveal a clear order of importance. Six features received similar scores: compact and lightweight (17\%, 49 responses), waterproof $(16 \%, 47$ responses), easy to use $(15 \%, 43$ responses), accuracy and signal $(19 \%, 54$ responses), battery life $(17 \%, 49$ responses $)$, and cost $(14 \%$, 41 responses). Two features were rarely chosen: memory (1\%, 2 responses) and connectivity (1\%, 3 responses). In total, 288 replies were recorded to this question.

Table 3: Frequency of GPS usage - total number of responses (Cnt) and percentages (Pctge).

\begin{tabular}{|l|r|r|}
\hline Frequency & Cnt & Pctge \\
\hline Only when I'm unsure & 17 & $25 \%$ \\
\hline At regular intervals & 22 & $33 \%$ \\
\hline Start of route & 0 & $0 \%$ \\
\hline Decision points & 10 & $15 \%$ \\
\hline Emergencies & 15 & $22 \%$ \\
\hline All the time & 2 & $3 \%$ \\
\hline Other & 1 & $1 \%$ \\
\hline Sum & $\mathbf{6 7}$ & $\mathbf{1 0 0 \%}$ \\
\hline
\end{tabular}

The next two questions aimed at identifying how often the GPS was used by those participants who had reported owning or having used such a device, and whether they used it for directions. Both questions allowed only for a single answer. The results for the first question are summarised in Table 3. A third of the participants (22 responses) reported using GPS devices at regular intervals, while $15 \%$ (ten responses) used them at decision points. A further $25 \%$ (17 participants) responded using it only when they are unsure about their current location; 22\% (15 responses) reported using only in case of an emergency. Almost no one used it continuously $(3 \%)$, following a different scheme $(1 \%$, one response) or at the start of the route ( $0 \%$, zero responses). Hence, almost $50 \%$ referred to their GPS device systematically (at regular intervals or at decision points) and nearly $50 \%$ of the users relied on it when they felt lost or in danger.
Table 4: Presentation of directions on GPS devices - total number of responses (Cnt) and percentages (Pctge).

\begin{tabular}{|l|r|r|}
\hline Presentation & Cnt & Pctge \\
\hline Text on screen & 14 & $21 \%$ \\
\hline Map image and marker & 38 & $57 \%$ \\
\hline Audio format & 0 & $0 \%$ \\
\hline Never used/not available & 7 & $10 \%$ \\
\hline Arrow or pointer & 5 & $7 \%$ \\
\hline Other & 3 & $4 \%$ \\
\hline Sum & $\mathbf{6 7}$ & $\mathbf{1 0 0 \%}$ \\
\hline
\end{tabular}

We then asked participants whether they use their GPS device for directions (rather than just for localisation), which an equal number of subjects (36\%, 24 responses) confirmed and denied. A further 19\% (13 participants) reported only requesting directions from their GPS unit when unsure; only $3 \%$ ( 2 respondents) stated that they rely on directions from their GPS device in low visibility conditions (e. g. low clouds, fog). $6 \%$ (4 replies) indicated other reasons as motivation for obtaining directions from their GPS device.

The following question were targeted at eliciting how directions were presented on GPS-equipped devices. Table 4 summarises our findings. The most frequent mode of presentation $(57 \%, 38$ responses) was the use of maps with markers, followed by textual output $(21 \%, 14$ responses). The other ways of presenting directions were much less common, with arrows and pointers used on $7 \%$ ( 5 responses) of the devices and other means on $4 \%$ (3 responses). Interestingly, none of the devices presented directions in an auditory format. $10 \%$ ( 7 responses) of the 67 participants responding to this question had never asked for directions or their device did not provide this feature.

The fourth set of questions in the questionnaire was again presented to all participants (whether they were GPS users or not), and was aimed at identifying potential landmarks in natural environments and what tools participants use when making navigational decisions. The first question in this set was an open question asking for three examples of natural landmarks, which participants see whilst in the countryside. Table 5 and Figure 3 summarise the responses we obtained.

The most frequently named landmarks, peaks and water courses accounted for more than $50 \%$ of the landmarks $(27 \%$, 144 responses and 26\%, 142 responses respectively). Further popular landmarks included woods ( $9 \%, 51$ responses), rocks (7\%, 38 responses), lochs (7\%, 36 responses), and gorges (5\%, 29 responses). All the other types of landmarks were named by less than $5 \%$ of the participants. Interestingly, man-made structures were listed by 22 participants, even though we had asked for examples of natural landmarks. In total, we recorded 547 responses for this question.

We then asked participants what tool they use most to verify their navigational decision when at a decision point. Only a single reply was permitted for this question. Almost two thirds of the participants $(63 \%, 120$ replies $)$ selected 'map' as their preferred means. Compass bearing (12\%, 23 replies) and route marker $(11 \%, 21$ replies) received considerably fewer votes as did GPS (7\%, 13 replies). $4 \%(8$ replies) of the participants said they did not verify their de- 
Table 5: Natural landmarks identified by participants: total number (Cnt) and percentages (Pctge).

\begin{tabular}{|l|r|r|}
\hline Natural Landmarks & Cnt & Pctge \\
\hline Peaks, summits, hills, knolls, slopes & 144 & $27 \%$ \\
\hline $\begin{array}{l}\text { Water courses, streams, stream junc- } \\
\text { tions, rivers, waterfalls }\end{array}$ & 142 & $26 \%$ \\
\hline Woods, forests, woodland & 51 & $9 \%$ \\
\hline Rocks, crags & 38 & $7 \%$ \\
\hline Lochs, lakes, tarns, bogs, ponds & 36 & $7 \%$ \\
\hline Gorges, gulleys, valleys, cols, spurs & 29 & $5 \%$ \\
\hline Man-made structures & 22 & $4 \%$ \\
\hline Edge, ridge, saddle, cliff & 17 & $3 \%$ \\
\hline Routes, pathways, trail markers, roads & 13 & $2 \%$ \\
\hline Field boundary, hedges, walls & 12 & $2 \%$ \\
\hline Land contours & 11 & $2 \%$ \\
\hline Trig points & 9 & $2 \%$ \\
\hline Beaches, coastline, sea & 7 & $1 \%$ \\
\hline Vegetation, plantation & 6 & $1 \%$ \\
\hline Sum & $\mathbf{5 4 7}$ & $\mathbf{1 0 0 \%}$ \\
\hline
\end{tabular}

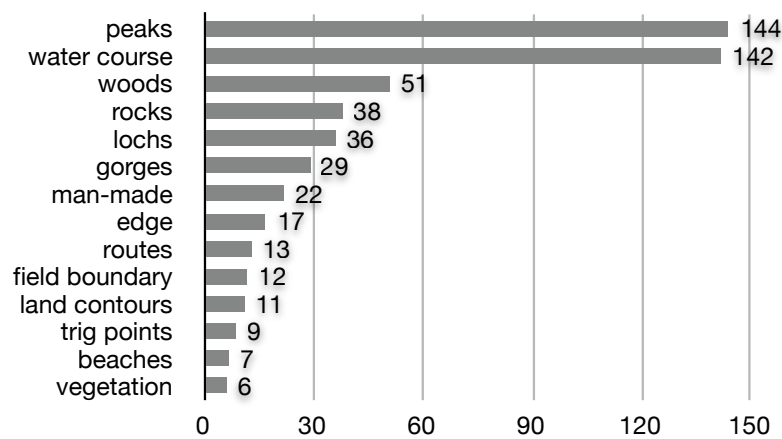

Figure 3: Natural landmarks identified by participants (number of responses).

cisions at all while only $1 \%$ (1 reply) named landmarks as their method of choice. A further 3\% (5 replies) used other means. In total, 191 participants responded to this question.

The following two questions were aimed at finding out how participants describe routes to others and what kind of descriptions they would find most useful. Table 6 and Figure 4 summarise the findings. In both cases, maps were most popular $-21 \%$ (153 responses) for giving directions and $30 \%$ (137 replies) for receiving them - followed by natural landmarks: $19 \%$ (143 responses) for giving directions and $20 \%$ (90 responses) for receiving them. The following three most selected means to convey directions were also the same for both giving and receiving directions but their relative ordered differed. Whereas in the case of giving directions, distances were rated highest $(14 \%, 107$ responses) followed by man-made structures (14\%, 106 responses) and OS grid references (11\%, 82 responses), the order for receiving directions was OS grid references (15\%, 67 responses) followed by distances $(11 \%, 49$ responses) and man-made structures $(9 \%, 41$ responses). The following three categories attracted a similar number of replies (although their relative order again varied between giving and receiving directions): compass bearings $(7 \%, 50$ responses for giving directions and $5 \%, 22$ responses for receiving them), guide books/online resources $(6 \%, 48$ responses for giving directions and $4 \%$ and 19 responses for receiving them), and photos (5\%, 38 responses for giving directions and 5\%, 23 responses for receiving). $2 \%$ of the participant listed other means as preferred means for giving directions (14 responses) or receiving them (9 responses). In total, 741 responses were recorded for giving directions and 457 for receiving them.

Table 6: Preferred means for giving and receiving directions (total number of responses (Cnt) and percentages (Pctge) - two replies permitted for giving directions and multiple replies permitted for receiving directions).

\begin{tabular}{|l|r|r||r|r|}
\hline & \multicolumn{2}{|c||}{ Giving } & \multicolumn{2}{|c|}{ Receiving } \\
\hline Means & Cnt & Pctge & Cnt & Pctge \\
\hline OS grid references & 82 & $11 \%$ & 67 & $15 \%$ \\
\hline map & 153 & $21 \%$ & 137 & $30 \%$ \\
\hline man-made structures & 106 & $14 \%$ & 41 & $9 \%$ \\
\hline distances & 107 & $14 \%$ & 49 & $11 \%$ \\
\hline natural landmarks & 143 & $19 \%$ & 90 & $20 \%$ \\
\hline $\begin{array}{l}\text { compass bearings, } \\
\text { cardinal directions }\end{array}$ & 50 & $7 \%$ & 22 & $5 \%$ \\
\hline $\begin{array}{l}\text { guide books, online } \\
\text { resources }\end{array}$ & 48 & $6 \%$ & 19 & $4 \%$ \\
\hline photos & 38 & $5 \%$ & 23 & $5 \%$ \\
\hline other & 14 & $2 \%$ & 9 & $2 \%$ \\
\hline Sum & $\mathbf{7 4 1}$ & $\mathbf{1 0 0 \%}$ & $\mathbf{4 5 7}$ & $\mathbf{1 0 0 \%}$ \\
\hline
\end{tabular}

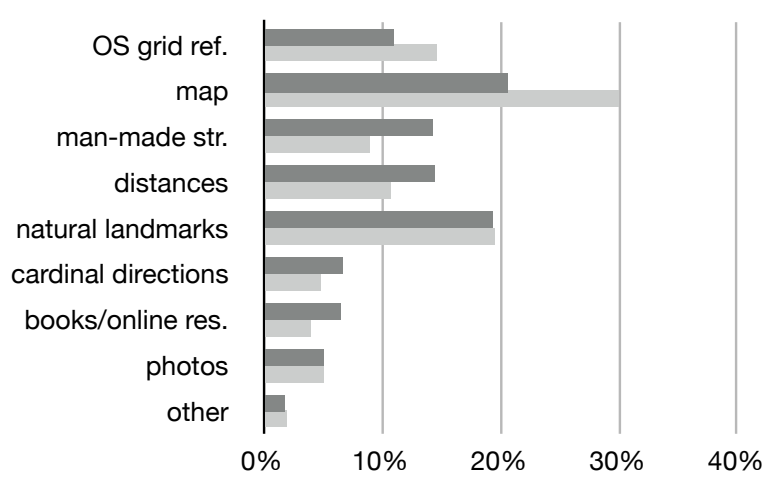

Figure 4: Preferred means to give directions (dark bars) and to receive directions (light bars) as percentages.

The last question in the fourth set was aimed at finding out how people document the routes they follow in the countryside. Table 7 and Figure 5 summarise the responses to this question (multiple replies were permitted). While a quarter of the replies (70 responses in total) indicate that quite a few people might not document a route at all or rely on their memory alone, the most popular means for documentation was taking photographs (30\%, 84 replies). Three 
further means were selected by a similar number of participants: notes $(13 \%, 37$ replies), lines on maps $(13 \%, 36$ replies) and online resources/blogs (10\%, 27 replies). The least popular options turned out to be GPS files $(4 \%, 10$ replies), postcards (3\%, 9 replies) and other means $(3 \%, 9$ replies). In total we recorded 282 replies for this question.

Table 7: Means used to document routes: total number (Cnt) and percentages (Pctge) - multiple replies were permitted.

\begin{tabular}{|l|r|r|}
\hline Means for documenting & Cnt & Pctge \\
\hline Notes & 37 & $13 \%$ \\
\hline Photos & 84 & $30 \%$ \\
\hline Lines on maps & 36 & $13 \%$ \\
\hline Postcards & 9 & $3 \%$ \\
\hline Online resources/blogs & 27 & $10 \%$ \\
\hline I don't/I rely on memory & 70 & $25 \%$ \\
\hline GPS files & 10 & $4 \%$ \\
\hline Other & 9 & $3 \%$ \\
\hline Sum & $\mathbf{2 8 2}$ & $\mathbf{1 0 0 \%}$ \\
\hline
\end{tabular}

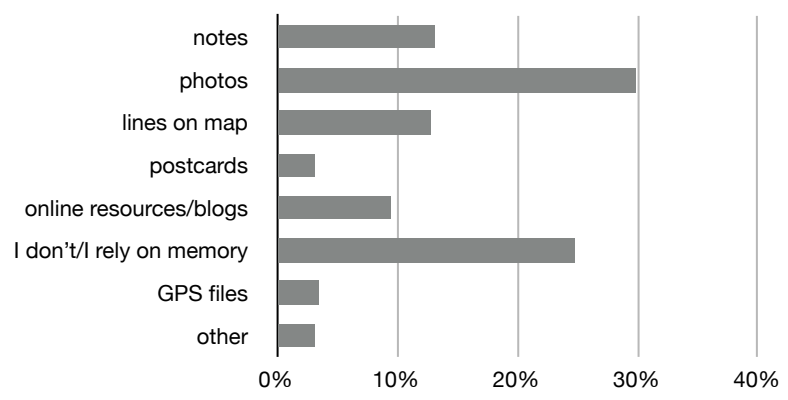

Figure 5: Means people use to document routes in natural environment (in percent) - multiple replies were permitted.

The final set of questions enquired after the age and gender of the participants, and asked them to rate their confidence in using online services on a $\mathrm{PC}$ or on a mobile phone, as well as their confidence in using an in-car sat-nav system. Figure 6 and 7 summarise the responses for these five questions. Roughly two thirds of the participants completing the whole survey were male $(68 \%, 119$ subjects), about a third was female (31\%, 54 subjects) and $2 \%$ (3 subjects) did not disclose their gender. In terms of age, $28 \%$ (50 subjects) declared being between 18 and 30 years old. 39\% (68 subjects) selected 31-45 as their age bracket and a further $27 \%$ (47 subjects) identified themselves as being between 46 and 60 years old. Only $4 \%$ ( 7 subjects) stated being between 61 and 75 ; no participant declared being older than 75 years. $2 \%$ (4 subjects) preferred not to disclose their age.

Since the questionnaire was distributed as an online questionnaire, it is not surprising that $73 \%$ (129) of the participants rated themselves as very confident in using online services on a desktop PC; a further $22 \%$ (39) selected the second highest confidence level. Only $3 \%$ (5 subjects) and $2 \%$ (3 subjects) rated their confidence as average and rather

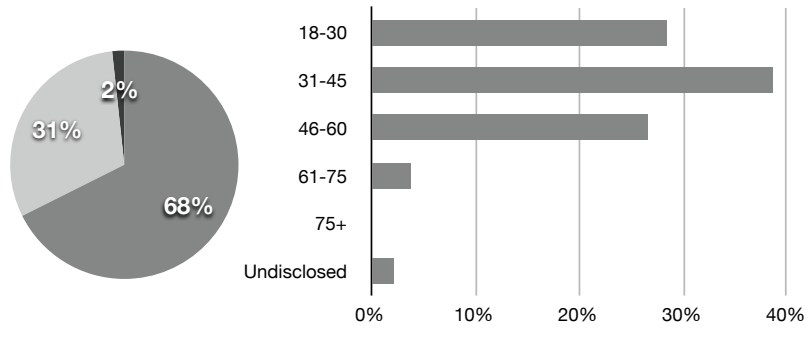

Figure 6: Gender distribution (pie chart; dark grey wedge: male, light grey: female, black: undisclosed) and age distribution (bar chart) of participants who filled out the entire questionnaire.

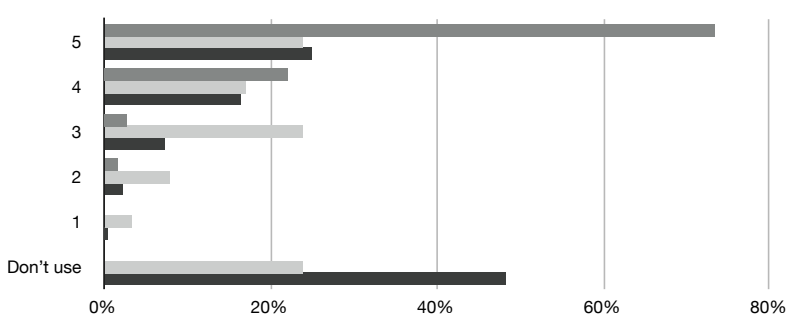

Figure 7: Self-assessed technology expertise (five point Likert scale, five corresponds to very confident, one to not confident at all); dark grey bars: desktop access to online services, light grey bars: mobile access to online services, black bars: in-car navigation systems.

low. No one selected the lowest confidence level or declared not using online services on a PC. This picture changed considerably, when we asked participants about their confidence in accessing such services from a mobile device. Only $24 \%$ (42 subjects) selected the highest confidence level, followed by $17 \%$ (30 subjects) picking the second highest category and a further $24 \%$ (42 subjects) rating their confidence as average. $8 \%$ ( 14 subjects) chose the second lowest level to describe their confidence in using online services on a mobile device and $3 \%$ (6 subjects) selected to lowest category. $24 \%$ (42 subjects) declared not using such services at all. When asked about their level of confidence in using an in-car navigation system, the overall picture again differed from both desktop and mobile access to online services. Almost half of the participant indicated that they do not use such systems $(48 \%, 85$ subjects $)$, and a further $1 \%$ (1 subject) and $2 \%$ (4 subjects) rated their confidence in using them as very low or low. $7 \%$ (13 participants) chose an average confidence level to describe their expertise. 25\% (44 subjects) described themselves as very confident users of in-car navigation systems and a further $16 \%$ (29 subjects) selected the second highest confidence level. In total, 176 participants replied to these three questions.

Unfortunately, the web service hosting the questionnaire returned the survey data in an aggregate format that did not allow for distinguishing between individual responses. This prevented us from analysing data more thoroughly, e. g. comparing results from different genders or age groups. 


\section{STUDY 2: LANDMARK-BASED NAVIGA- TION SUPPORT}

As the results from the questionnaire study indicated that quite a few people used landmarks for navigation, we decided to evaluate their use for navigation support on mobile devices further. We considered conducting a field study but there were two issues with the particular scenario and application we intended to test that made conducting the study in situ difficult. Participants would have to be taken to a somewhat remote location without much infrastructure (e.g. in terms of guaranteeing their comfort and safety in case of an emergency), and they would be exposed to (potentially) large variations of environmental conditions/and or contexts. In particular, the weather as well as the visibility can vary widely and quickly in the region, which could not only influence results but might also be potential uncomfortable or even hazardous to participants.

In order to circumvent these issues we decided to use a modified version of the immersive video approach that was applied in previous studies investigating the use of mobile navigation support in urban environments [18, 19]. This approach is based on the idea of immersing participants in video footage that was captured by multiple cameras and played back on multiple screens. In the example discussed by Singh et al. [18], they used a three sided CAVE system (a visualisation environment designed for virtual reality applications). Participants were placed in front of a roughly semicircular arrangement of three large screens so that their entire field of vision was filled with the video footage.

\subsection{Design}

Our physical set-up was similar to the one described in [18] (three-wall CAVE in roughly semicircular arrangement) but instead of video footage, we used panoramic photographs of decision points along a sample route through the countryside. This approach resulted in a realistic visual simulation of the natural environments while not having to expose participants to the potential discomfort of bad weather conditions. Using photographs instead of video footage had the benefit of creating an image of the landscape at the highest possible resolution and clarity. (Video footage is often subject to compression artefacts.) A further advantage (shared with immersive video) was fine-grained control of the context, i. e. every participant experienced the different locations in exactly the same way (same weather conditions, same time of the year, same time of the day, same point of view). This was particularly important in our case as natural landmarks can change significantly depending on the season, weather conditions or time of the day. In order to realistically assess the use of landmarks on mobile devices, landmarks were shown to the participants on a mobile device. Our main goal in conducting this study was to obtain qualitative feedback from participants regarding whether or not they considered landmarks shown on mobile devices useful for navigation in the countryside. We were also interested in which natural landmarks they would (not) use and why.

\subsection{Method}

Material. We created a set of 16 panoramic photographs from a series of photographs taken with a mid-range digital camera on a trip to the local countryside. Three photos were taken per location, and stitched together using a com-

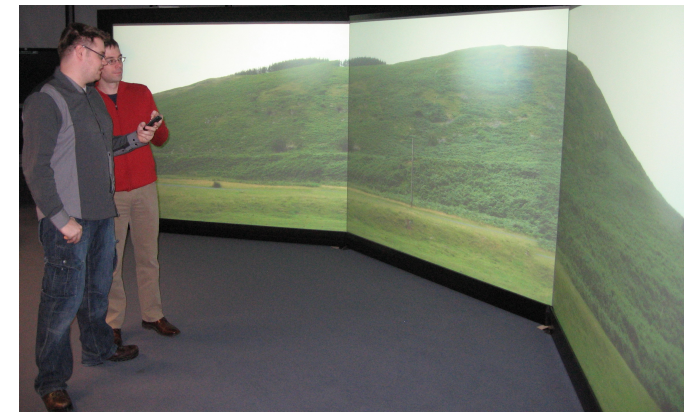

Figure 8: Set-up for interviews using immersive panoramic photos: participant and experimenter discuss landmark shown on mobile device while a high-resolution panoramic photo of the corresponding decision point is shown on the large-scale display.

mercial tool to create one seamless panorama per location. For each of the locations, we also created a set of landmark photographs; landmarks were selected according to the list we had obtained in the questionnaire (see Table 5). The panoramic photographs were shown on a three-sided CAVE system shown in Figure 8. Each wall is approximately $2.5 \mathrm{~m}$ wide and $2 \mathrm{~m}$ tall and has a resolution of $1280 \times 1024$ pixel, resulting in an overall resolution of $3840 \times 1024$ pixel. When selecting landmarks for a location, we varied type and 'obviousness', i. e. in a location where two trees were growing near a river, we would pick either the river or the tree as a landmark. We also varied the angle from which landmark photographs were taken, i. e. the direction from which a landmark was photographed did not always coincide with the direction from which the panoramic photograph was shot. The landmark photographs were shown to subjects on a PDA with a 3.5 " colour screen $(320 \times 240$ pixel $)$; Figure 9 shows an example photo of a landmark. We used one photograph of a landmark per location in each trial. The photo sets varied slightly between trials (i. e. we did not always use the same landmark in each trial). We adapted a set of simple PHP scripts to control three instances of an open source media player, which in turn rendered the left, the middle and the right part of a panoramic image on the corresponding screen, and which was synchronised with the landmark displayed on the mobile device.

Participants. We recruited three participants from local clubs engaging in outdoor/countryside activities via postings on a number of mailing lists. While the small number of subjects limits the statistical analysis of the results, we considered it sufficient due to the exploratory nature of our work and our focus on qualitative results.

Procedure. Participant received a short briefing prior to the experiment, explaining the overall structure of the experiment and informing them about the audio recording. Figure 8 shows the physical set-up of the immersive panorama study in the CAVE. Participants stood about three meters away from the displays, with the experimenter next to them taking notes and recording the interview. An assistant was operating the software controlling the large display, and was switching between the panoramic photographs on a hand signal given by the experimenter. Participants were holding the PDA while being interviewed by the experimenter in a semistructured way. For each location, we asked participants to 


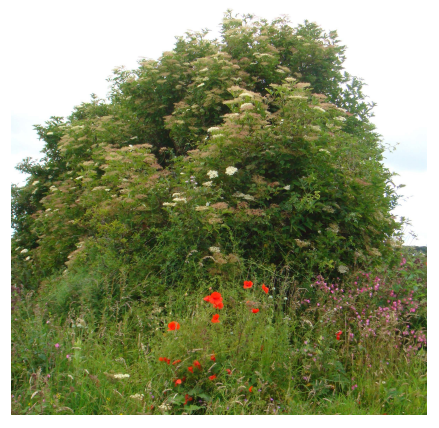

Figure 9: Example photograph of a landmark show to participants on a mobile device.

describe the scene shown on the panoramic photograph and whether they could identify the landmark shown on the PDA on the large screen. We also queried them about the feasibility of the landmark for navigation purposes. If they felt is was not so well suited, we asked them to pick a landmark from the panoramic photograph, which they thought was most useful for navigation purposes. Once discussion about a location was finished, the next location was displayed on the large display. A simple PHP script was used to record the amount of time spent discussing each location.

\subsection{Results}

The analysis of the time taken per location/landmark proved to be somewhat inconclusive (see Table 8). Participants spent between 22 and 47 minutes on the entire interview, and average/minimum/maximum times per location/landmark pair also varied considerably. The same was true when looking at the relative distribution of times; participants spent different amounts of time on discussing each location. Only in three instances (landmark/location four, five and seven) was there a slight correlation in the sense that every participant spent an above average amount of time talking about these locations. These three landmarks showed vegetation (trees, shrubs, patches of coloured ground vegetation) and were identified as less well suitable by the participants but there were other landmarks in the set for which the same was true. One location/landmark pair (number 15) was discussed for a relatively short time (either shortest or second shortest). The corresponding landmark photograph showed a stately building on a hill and all participants felt that this was a 'good' landmark but again there were other man-made landmarks in the set (e.g. a viaduct and a ruined castle), which they approved of as well. The times per landmark from the beginning to the end of a study varied for each subject but there was a tendency for more discussion during the first half of the study.

Table 8: Time taken per location/landmark pair.

\begin{tabular}{|l|r|r|r|}
\hline & \multicolumn{3}{|c|}{ Participant } \\
\hline Time per location & E1 & E2 & E3 \\
\hline Average & $95 \mathrm{~s}$ & $115 \mathrm{~s}$ & $202 \mathrm{~s}$ \\
\hline Minimum & $55 \mathrm{~s}$ & $77 \mathrm{~s}$ & $51 \mathrm{~s}$ \\
\hline Maximum & $173 \mathrm{~s}$ & $183 \mathrm{~s}$ & $332 \mathrm{~s}$ \\
\hline Total time of interview & $22^{\prime} 16^{\prime \prime}$ & $26^{\prime} 56^{\prime \prime}$ & $47^{\prime} 8^{\prime \prime}$ \\
\hline
\end{tabular}

An analysis of the interview transcripts revealed that every participant was able to identify all landmarks provided on the mobile device, i. e. they were able to spot them on the corresponding panorama - even when the landmark photographs were taken from a different location than the panorama itself. Overall, the three participants rejected the proposed landmark 26 times and accepted it 21 times (on one occasion an image did not load properly and was skipped). Of those landmarks that were the same in each participant's sets, there was general agreement in seven cases (i. e. they either all rejected or accepted the proposed landmark) and disagreement in three cases. (The remaining six photographs in each set varied between sets.) Four of the seven landmarks, which everyone judged in the same way, were seen as being suitable as landmarks. The corresponding photographs showed: a patch of ground vegetation that visually differed from the ground vegetation around it, a road and some pylons, a church and a stately building. In terms of the landmarks suggested by the interviewees, there was only one case where they all suggested the same object as a landmark (a junction between a road and a path); in all other cases, different landmarks were suggested by each participant.

A further observation from the study relates to the way in which people referred to the panoramic photographs. At the beginning of a trial, participants clearly distanced themselves from the image shown on the large screens. For example, E1 referred to the first panorama by saying "I would describe it as, erm, a hill with an outcrop of rocks on it." E2 describe the second panorama as "It's a woodland landscape [...]", and E3 started discussing the first panorama by saying "So we can see in this is basically a hillside covered with ferns." (emphasis added by the authors). Later in trial, the way they were describing the panoramic images, often referred to the images as if they were in the actual location. For example, E1 referred to the second panorama by saying "we are in a wood" and E2 started discussing the fourth panorama saying "We appear to be in another type of valley [...]". E3 referred to the last panorama by saying "We are in a meadow with some clover and buttercups [...]". E3 in particular also frequently described the scene by self-locating first with respect to the landscape shown on the panoramic photographs. (We also observed this kind of self-localisation for the other two participants but less frequently than in the case of E3.) Examples for this kind of utterances include:

- "we are looking from what appears to be a grassy flat area, over a road [...]" (second panorama)

- "looking down a valley, hills on either side" (third panorama)

- "A river in the foreground running from left to right, on $m y$ shore there is a grassy pasture like landscape, [...]" (seventh panorama, emphasis by author)

- "looking down over a valley from a slightly elevated point" (eighth panorama)

- "standing at a crossroads where, there is a road to the left [...]" (ninth panorama)

\section{DISCUSSION}

The data we gathered in both studies indicates that landmarks seem to be an important means by which people navigate in the countryside, despite most of our participants 
relying on traditional methods such as maps and compass bearings. Even though almost no one listed landmarks as their preferred means of navigation, landmarks received the second highest rating for both giving and receiving directions. This view was supported by the behaviour of the participants in the second study, which readily engaged with and used landmarks both on the large display and the mobile device. One participant was going as far as stating "How do you not navigate using landmarks?".

Regarding the types of landmarks used, peaks and water courses were the most frequently named natural landmarks. This was somewhat supported by the participants in our second study, which would often pick a water course as a suggested/preferred landmark if it was present in a panoramic image. While there were only very few 'peaks' depicted in the landscape photographs, when there was one, it was picked as a preferred landmark quite frequently. An explanation for the strong preference for hills and water courses may be that the former is highly visible from afar and the latter one fairly unambiguous and spatially extended. Both may also constitute an obstacle that is difficult to overcome, and their appearance varies very little according to the time of the year. Clearly, the type and relative importance of different types of landmarks depends on the region. The landmarks we collected relate to a wet and rather flat landscape with mostly low vegetation. Landmarks for use in other areas (e.g. mountainside) would likely be different.

A further observation regarding the types of landmarks relates to the importance of man-made structures. Even though we asked for natural landmarks in the questionnaire, man-made structures and routes were listed by 35 people. In the immersive video study, three out of four landmarks that were unanimously accepted also were man-made structures. In addition, participants in the second study referred to paths quite frequently when suggesting landmarks. Despite the relevance of man-made structures, there are obviously differences between landmarks used for urban navigation and navigation in the countryside (see also Table 5). For example, features of the landscape such as raised/lowered terrain or watercourses are very prominent for use in the countryside, whereas for urban navigation they play a much smaller (if any) role. Furthermore, while streets and the street network are key elements of urban navigation, paths are of lesser importance in the countryside (only $2 \%$ mentioned them in the questionnaire).

Combining the results and observations from both studies, there are some solid indications that it might be feasible to use photographs of natural landmarks on a mobile device for navigation in the wild. Each participant in the immersive panorama study was able to identify/spot all the landmarks that were shown on the mobile device, including those that had been taken from a different location than the panoramic photograph itself. While the identification of the most suitable landmark for a given decision points will require further research, there was a considerable level of agreement amongst the participants of the second study what does and what doesn't constitute a good landmark for use on a mobile device.

A further point contributing to the feasibility of landmarkbased mobile navigation support in the wild was the fact that taking photographs was the most popular way of documenting a trip to the countryside. Arguably, the chance of someone photographing a landmark rather than some in- distinct part of the landscape is fairly high. Provided these photos would be geo-tagged, they could contribute to creating a community-driven database of landmark photographs for use by a mobile guide, which would help to address the issue of how to collect all the photographic material to make a navigation service feasible. Unlike similar services, which have been designed for urban areas such Street View (http://maps.google.com/help/maps/streetview/), a service for natural environments would have to take into account time of the day and time of the year to account for seasonal differences such as shrubs being in bloom or rivers being dried up in summer (e. g. by analysing the time stamp associated with most digital photographs).

The results we obtained also highlight a potential issue that needs to be overcome when developing and deploying a landmark-based navigation service for use on mobile devices in the countryside. Most participants have been relying on their preferred means of navigation for a long time, mostly maps or maps and compass, despite GPS devices being readily available for several years now. In addition, while all participants in the questionnaire study were highly confident in using online services from a desktop, considerably fewer had done so on mobile devices, with a large percentage indicated they had never done so. One promising option to address these issues might be to complement rather than replace the navigation methods currently used by most of our participants, e.g., by augmenting maps with photographs of landmarks to support navigation. Such an approach has been shown to work well when applied to urban settings [16].

Analysing the outcomes of the second study, there are some interesting observations regarding the new evaluation technique we employed. While panoramic photographs lack the 'liveliness' and sound provided by immersive video, they depict a location at very high resolution. When placed at the distance from the screen where we placed participants, it is not possible to make out individual pixels, whereas when showing video, some pixelation would be visible. The benefit of this was apparent on several occasions, when participants were able to identify details such as the type of a distant tree or a gate largely hidden by some vegetation, which would have been difficult to make out at lower resolution.

In addition, we gathered some evidence that people do immerse themselves into the panoramic photograph even though the image is static. All participants frequently referred to the image as if there were in the actual location rather than in front of a screen showing a photograph. We also observed on several occasions that people would describe their location with respect to the landscape shown on the screen. Although further research is needed in this respect, these findings indicate that immersive photographs shown on a large screen may constitute an interesting middle ground between field studies and lab-based studies. Obviously, there are some trade-offs compared to a real field study (such as not physically moving to get to a different location) but the panoramas seem to be inductive to subjects feeling as if there in the actual location while providing experimenters with full control over the context of use.

\section{CONCLUSION}

In this paper, we reported on two studies exploring the use of landmarks for mobile navigation support in natural environments. Our aim was to shed light on an area in pedestrian navigation support, which so far has received very little 
attention. In doing so, we were able to make three main contributions. Firstly, we collected some initial empirical data on how people navigate in the countryside, and we identified what kind of natural landmarks they use. Secondly, we gathered initial evidence that landmark-based navigation on mobile devices may indeed be feasible for use in natural environments. Thirdly, our results support the conjecture that immersive panoramic photographs may constitute a useful middle ground between field studies and lab-based studies when evaluating mobile systems.

Based on our findings, there are several promising directions for future research. It would be interesting to repeat a similar set of studies with a larger number of participants and for different regions in order to identify whether and how navigation behaviour and landmarks used differ. This would also contribute to identifying key features of (natural) landmarks in natural environments. A further obvious direction would be to develop a working prototype of a guide for natural environments that makes use of landmarks, and to gather feedback from users. Finally, the immersive panorama approach has shown some promise in terms of evaluating mobile systems. We therefore plan to conduct a series of comparison studies, where we analyse the properties, drawbacks and advantages of this method compared to other commonly used evaluation methods for mobile devices.

\section{REFERENCES}

[1] M. Arikawa, S. Konomi, and K. Ohnishi. Navitime: Supporting pedestrian navigation in the real world. IEEE Pervasive Computing, 6(3):21-29, 2007.

[2] J. Baus, C. Kray, and K. Cheverst. A survey of map-based mobile guides. In L. Meng, A. Zipf, and T. Reichenbacher, editors, Map-based Mobile Services, pages 197-216. Springer, Berlin, Heidelberg, New York, 2005

[3] N. J. Bidwell, C. Lueg, and J. Axup. The territory is the map: designing navigational aids. In CHINZ '05: Proceedings of the 6th ACM SIGCHI New Zealand chapter's international conference on Computer-human interaction, pages 91-100, New York, NY, USA, 2005. ACM.

[4] A. Butz, J. Baus, A. Krüger, and M. Lohse. A hybrid indoor navigation system. In Proceedings of IUI 2001, pages 25-32, New York, 2001. ACM Press.

[5] K. Cheverst, N. Davies, K. Mitchell, and A. Friday. Experiences of developing and deploying a context-aware tourist guide: the GUIDE project. In Mobile Computing and Networking, pages 20-31, Boston, U.S.A., 2000.

[6] N. Davies, K. Cheverst, A. Dix, and A. Hesse. Understanding the role of image recognition in mobile tour guides. In MobileHCI '05: Proceedings of the 7th international conference on Human computer interaction with mobile devices $\mathscr{E}$ services, pages 191-198, New York, NY, USA, 2005. ACM.

[7] C. Düpmeier and M. Ruchter. User interface architecture of a mobile guide for exploring the wild. In 3rd International Workshop on "HCI in Mobile Guides" at Mobile HCI'04, Glasgow, Scotland, September 132004.

[8] J. Goodman, P. Gray, K. Khammampad, and S. Brewster. Using landmarks to support older people in navigation. In Proceedings of Mobile HCI 2004 , pages 38-48, Heidelberg, Berlin, New York, 2004. Springer.

[9] S. Holland, D. R. Morse, and H. Gedenryd. Audiogps: Spatial audio navigation with a minimal attention interface. Personal and Ubiquitous Computing, 6(4):253-259, 2002.

[10] C. Hölscher, T. Meilinger, G. Vrachliotis, M. Brösamle, and M. Knauff. Up the down staircase: Wayfinding strategies in multi-level buildings. Journal of Environmental Psychology, 26(4):284-299, 2006.

[11] C. Kray, K. Laakso, C. Elting, and V. Coors. Presenting route instructions on mobile devices. In W. L. Johnson, E. André, and J. Domingue, editors, Proceedings of IUI 03, pages 117-124, Miami Beach, FL, 2003. ACM Press.

[12] C. Nothegger, S. Winter, and M. Raubal. Selection of salient features for route directions. Spatial Cognition and Computation, 4(2):113-136, 2004.

[13] G. Pospischil, M. Umlauft, and E. Michlmayr. Designing LoL@, a Mobile Tourist Guide for UMTS. In F. Paterno, editor, Proceedings of Mobile Human-Computer Interaction 2002, pages 140-154, Berlin, Heidelberg, New York, 2002. Springer.

[14] M. Raubal and S. Winter. Enriching wayfinding instructions with local landmarks. In Geographic Information Science, pages 243-259, Heidelberg, Berlin, New York, 2002. Springer.

[15] Y. Rogers, S. Price, G. Fitzpatrick, R. Fleck, E. Harris, H. Smith, C. Randell, H. Muller, C. O'Malley, D. Stanton, M. Thompson, and M. Weal. Ambient wood: designing new forms of digital augmentation for learning outdoors. In IDC' 04 : Proceedings of the 2004 conference on Interaction design and children, pages 3-10, New York, NY, USA, 2004. ACM.

[16] M. Rohs, J. Schöning, M. Raubal, G. Essl, and A. Krüger. Map navigation with mobile devices: virtual versus physical movement with and without visual context. In ICMI '0\%: Proceedings of the 9th international conference on Multimodal interfaces, pages 146-153, New York, NY, USA, 2007. ACM.

[17] E. Rukzio, A. Schmidt, and A. Krüger. The rotating compass: a novel interaction technique for mobile navigation. In $C H I$ '05: $C H I$ '05 extended abstracts on Human factors in computing systems, pages 1761-1764, New York, NY, USA, 2005. ACM.

[18] P. Singh, H. N. Ha, Z. Kuang, P. Olivier, C. Kray, P. Blythe, and P. James. Immersive video as a rapid prototyping and evaluation tool for mobile and ambient applications. In MobileHCI '06: Proceedings of the 8th conference on Human-computer interaction with mobile devices and services, pages 264-264, New York, NY, USA, 2006. ACM Press.

[19] P. Singh, H. N. Ha, P. Olivier, C. Kray, Z. Kuang, A. W. Guo, P. Blythe, and P. James. Rapid prototyping and evaluation of intelligent environments using immersive video. In Proceedings of MODIE workshop at Mobile HCI'06, Espoo, Finland, 12-15 September 2006. 